\title{
Self-Assembling Cyclic Peptides: Molecular Dynamics Studies of Dimers in Polar and Nonpolar Solvents ${ }^{\dagger}$
}

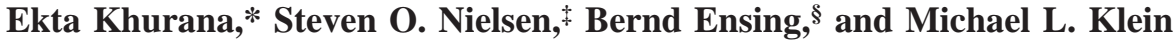 \\ Center for Molecular Modeling and Department of Chemistry, University of Pennsylvania, \\ Philadelphia, Pennsylvania 19104-6323
}

Received: December 22, 2005; In Final Form: March 17, 2006

\begin{abstract}
The self-assembly of cyclic D,L- $\alpha$-peptides into hollow nanotubes is a crucial mechanistic step in their application as antibacterial and drug-delivery agents. To understand this process, molecular dynamics (MD) simulations were performed on dimers of cyclic peptides formed from cyclo [(-L-Trp-D-N-MeLeu- $\left.)_{4}-\right]_{2}$ and cyclo [(-L-Trp-D-Leu- $\left.)_{4}-\right]_{2}$ subunits in nonpolar (nonane) and polar (water) solvent. The dimers were observed to be stable only in nonpolar solvent over the full $10 \mathrm{~ns}$ length of the MD trajectory. The behavior of the dimers in different solvents is rationalized in terms of the intersubunit hydrogen bonding, hydrogen bonding with the solvent, and planarity of the rings. It is shown that the $\varphi$ and $\psi$ dihedral angles of a single uncapped ring in nonane lie in the $\beta$-sheet region of the Ramachandran plot, and the ring stays in a flat conformation. Steered MD (SMD) simulations based on Jarzynski's equality were performed to obtain the potential of mean force as a function of the distance between the two rings of the capped dimer in nonane. It is also shown that a single peptide subunit prefers to reside close to the nonane/water interface rather than in bulk solvent because of the amphiphilic character of the peptide ring. The present MD results build the foundation for using MD simulations to study the mechanism of the formation of cyclic peptide nanotubes in lipid bilayers.
\end{abstract}

\section{Introduction}

Ghadiri and co-workers have designed remarkable synthetic nanotubes, which are formed by the self-assembly of cyclic peptides with an even number of alternating D- and L-amino acid residues. The cyclic peptides in question adopt a planar ring conformation and stack, under favorable conditions, to form hydrogen-bonded nanotubes, which can act as selective antibacterial agents. Moreover, the cyclic D,L- $\alpha$-peptide building blocks are proteolytically stable, easy to synthesize, and can be derived from a potentially vast membrane-active sequence space. ${ }^{1}$ Such self-assembling peptide nanotubes have been studied experimentally by high-resolution X-ray crystallography, cryoelectron microscopy, electron diffraction, and Fourier transform infrared (FT-IR) spectroscopy, ${ }^{2-7}$ as well as theoretically by $\mathrm{ab}$ initio and semiempirical approaches. ${ }^{8-10}$ Molecular dynamics (MD) simulations focusing on the structure and dynamics of pre-assembled nanotubes have also been performed. ${ }^{11,12}$ The computational studies have thrown light on the structure of these nanotubes and their action as ion channels.

In an ideal supramolecular nanotube assembly, rings stack through backbone-backbone $\beta$-sheet-like hydrogen bonding. The individual peptide rings adopt a planar conformation in which all amide backbone functionalities reside approximately perpendicular to the plane of the ring structure. Steric considerations and the alternating amino acid configuration force all the peptide side chains to lie on the outside of the ensemble,

\footnotetext{
† Part of the special issue "Robert J. Silbey Festschrift".

* Corresponding author.

Present address: Department of Chemistry, University of Texas at Dallas, P.O. Box 830688, Richardson TX 75083-0688.

$\S$ Present address: Department of Chemistry and Applied Biosciences, ETH Zurich USI-Campus, Via Giuseppe Buffi 13, Lugano, CH-6900 Switzerland
}

which results in a hollow tubular core. ${ }^{6}$ The side chains influence the self-assembly process and functionality of the nanotubes. If the peptide subunits have appropriate hydrophobic surface characteristics, they undergo rapid partitioning into lipid bilayers and spontaneously self-assemble into ion-transport-competent membrane channel structures. These nanotubes then display good channel-mediated ion-transport activity with rates exceeding $10^{7}$ ions per second. ${ }^{4}$

Details of the assembly process of these tubes and their mode of action as antibacterial agents are lacking. Although it is thought that peptide nanotubes operating through the carpetlike mechanism would have the greatest potential for membrane discrimination because of electrostatic interactions with various membrane constituents, ${ }^{1}$ an understanding of the self-assembly process will help determine what factors favor this mode of action. The goal of the present study is therefore to understand elements of the self-assembly process generating the cyclic peptide nanotubes. To this end, classical MD simulations have been performed on cyclic peptide dimers composed of two cyclo [(-L-Trp-D-N-RLeu- $\left.)_{4}\right]_{2}$ subunits $\left(\mathrm{R}=\mathrm{H}, \mathrm{CH}_{3}\right)$. In the case of $\mathrm{R}=\mathrm{CH}_{3}$, via selective methylation of backbone amide nitrogen functionalities, the cyclic peptide subunit is devoid of hydrogenbond donation from one face of the ring, so that it is predisposed toward an antiparallel stacked, cylindrical dimer. The "capped" dimer, which contains the key fundamental repeating structural motif of longer peptide nanotubes, has been studied experimentally. ${ }^{5}$ The $(\mathrm{R}=\mathrm{H})$ model dimer system, with uncapped nitrogen ends, is also studied. This subunit, which is known to self-assemble in a lipid bilayer environment and acts as an antibacterial agent, will be important in future studies. ${ }^{3,4,13,14}$ Besides their behavior in a lipid bilayer environment, cyclic rings made of alternating L-Trp and D-Leu residues have also been shown to self-assemble in organosulfur self-assembled monolayers on gold film. ${ }^{15}$ 
The nanotubes formed by the self-assembly of cyclic peptide subunits in a lipid bilayer are known to possess a $\beta$-sheet structure from various experimental studies. Here we show that the $\varphi$ and $\psi$ dihedral angles of a single uncapped peptide ring in a nonpolar solvent also lie in the $\beta$-sheet region of the Ramachandran plot.

The thermodynamic basis for the self-assembly process comes from an experimental study performed on a cyclic peptide with a selectively $\mathrm{N}$-methylated backbone that was shown to form discrete soluble cylindrical dimers in $\mathrm{CCl}_{4}$ and $\mathrm{CDCl}_{3}{ }^{5}$ It was found that the peptide subunit cyclo [(-L-Phe-D-N-MeAla- $\left.)_{4}-\right]$ adopts a flat-ring-shaped conformation, which energetically favors ring stacking and intermolecular hydrogen-bonding interactions over the nonassembled form by $4.0-5.6 \mathrm{kcal} \mathrm{mol}^{-1}$, depending on the solvent. The free energy of the dimerization process is accessible via steered MD (SMD) simulations using Crooks theorem ${ }^{16,17}$ in the form of the Jarzynski equality. ${ }^{18-20}$

Understanding the behavior of a single peptide subunit at the hydrophobic/hydrophilic interface is crucial to the understanding of the mechanism of formation of a nanotube in a hydrated lipid bilayer. Along these lines, we conducted MD simulations of a single uncapped peptide subunit in a nonane/water interfacial system. Irrespective of the initial starting configuration, the amphiphilic peptide subunit prefers to reside close to the interface in the nonane phase. However, when extrapolating this observation to the hydrated lipid bilayer, it should be kept in mind that the hydrophobic/hydrophilic interface in a bilayer is much more complex and broader than a simple nonane/water interface.

The results of the present study on dimerization indicate that the nanotube assembly probably takes place in the hydrophobic region of the hydrated lipid bilayer. This study in a hydrophobic and a hydrophilic solvent builds the foundation for an understanding of the tube elongation process in a heterogeneous membrane environment.

\section{Simulation Details}

A hydrated lipid bilayer consists of two sheets of amphiphilic phospholipids oriented tail-to-tail so that the hydrocarbon region is sequestered from water exposure by the hydrophilic heads. Before attempting to study peptide nanotube self-assembly in a lipid bilayer, it was thought prudent to begin with studies of the cyclic peptides in pure solvents that mimic the two environments of the bilayer. Nonane and water were used as the hydrophobic and hydrophilic solvents, respectively.

The starting peptide coordinates for the MD simulations were taken from the X-ray crystallographic coordinates provided by M. Reza Ghadiri (private communication). The X-ray coordinates were for the cyclo [(-L-Gln-D-Leu- $\left.)_{4}-\right]_{2}$ dimer in an antiparallel $\beta$-sheet structure. The L-Gln side chains were changed to L-Trp side chains using the ACCELRYS visualization package Insight II. Alternating D- and L-conformations of the backbone constrain the $\beta$-sheet structure so that only homochiral residues can form the cross-strand $\beta$-sheet nearest pairs. ${ }^{21}$ MD simulations were carried out using the program NAMD version $2.5 \mathrm{~b} 1^{22}$ and the CHARMM force field. ${ }^{23}$

Periodic boundary conditions were used in three dimensions. The Nosé-Hoover Langevin piston pressure control method was used to maintain the pressure of the cell at 1 atm during isobaric simulation runs, and, in all the simulations, Langevin dynamics was used to control the temperature at $300 \mathrm{~K}$. A time step of 2 fs was used. Nonbonded interactions were calculated every time step, and full electrostatic interactions were calculated every two time steps. Bonds between hydrogens and heavy atoms were constrained to their equilibrium value by means of the SHAKE/ RATTLE algorithm. ${ }^{24,25}$ Long-range electrostatic forces were taken into account using the particle mesh Ewald approach with a real space cutoff of $12 \AA{ }^{26,27}$ The water molecules were described using the TIP3P model. ${ }^{28}$

The following four MD simulations (designated by Roman numerals I-IV) were performed on the cyclic peptide dimers: (I) the capped dimer cyclo $\left[(-\mathrm{L}-\mathrm{Trp}-\mathrm{D}-\mathrm{N}-\mathrm{MeLeu}-)_{4}-\right]_{2}$ in 3024 water molecules; (II) the uncapped dimer cyclo [(-L-TrpD-Leu- $\left.)_{4}-\right]_{2}$ in 3031 water molecules; (III) the capped dimer cyclo [(-L-Trp-D-N-MeLeu- $\left.)_{4}\right]_{2}$ in 300 nonane molecules; and (IV) the uncapped dimer cyclo [(-L-Trp-D-Leu- $\left.)_{4}-\right]_{2}$ in 293 nonane molecules. A box consisting of 316 molecules of nonane was created and equilibrated for $1 \mathrm{~ns}$ using the isothermalisobaric (NPT) ensemble and allowing isotropic cell fluctuations (maintaining a constant ratio of $x: y: z$ ). The box dimensions converged to $45.75 \times 45.75 \times 45.75 \AA$ after about 100 ps. The respective dimers were then added to this box, resulting in the removal of a few nonane molecules to avoid overlap.

All the systems for cases I-IV were equilibrated for $1 \mathrm{~ns}$ by constraining the $\mathrm{C} \alpha$ atoms of the backbone to their initial positions, using the NPT ensemble, and allowing isotropic cell fluctuations. This allowed equilibration of the side chains and the solvent molecules. The box sizes converged after a few picoseconds. After $1 \mathrm{~ns}$ of equilibration, the constraints on the $\mathrm{C} \alpha$ atoms were removed, and the NVT ensemble was used for production runs. The box dimensions for cases I and II were $46 \times 46 \times 44 \AA$, while, for case III the box dimensions were $45.5 \times 45.5 \times 45.5 \AA$, and for case IV they were $45 \times 45 \times$ $45 \AA$. In addition, we conducted an MD simulation of one uncapped ring in 303 nonane molecules for $3 \mathrm{~ns}$ using the NPT ensemble with isotropic cell fluctuations.

The free energy of dimerization was studied for the capped dimer in nonane (case III) in the following manner. The $(x, y, z)$ coordinates of the center of mass of one ring were kept fixed. The center of mass of the second ring was pulled away from that of the first ring in the direction parallel to the intersubunit hydrogen bonding $(z)$ direction. Force was applied to all the atoms of the rings to constrain the respective centers of mass. The $(x, y)$ coordinates of the pulled ring were not constrained. Initial conditions were drawn from constrained MD runs of 1 $\mathrm{ns}$ at separation distances of $\sim 6.5,7.5,8.5, \ldots$, and $12.5 \AA$ between the $z$ coordinates of the two rings. Every $50 \mathrm{ps,} \mathrm{a} \mathrm{frame}$ was saved to initiate a total of 20 SMD pulling runs for each separation distance. In this fashion, a total of 140 pulling runs corresponding to pulling for $1 \AA$ each were launched, resulting in a total pulling distance of $7 \AA$. To join the different freeenergy profiles at the overlapping values of $z$, the curve that had better sampling (more data points) for that region was chosen. At the maximum separation distance of $13.5 \AA$, the backbones of the two rings are not interacting with each other. In every case, the reaction coordinate was linked to the pulling coordinate with a harmonic spring of stiffness $250 \mathrm{kcal} / \mathrm{mol} / \AA^{2}$ (17369.75 pN $\AA$ ). The force constant was chosen such that it was large enough to ensure small deviation of the reaction coordinate from the constraint position. The pulling velocity in each case was $5 \AA / n s$. The free energy calculation was based on Jarzynski's equality and the stiff-spring approximation described by Park and Schulten. ${ }^{29}$ The free energy was calculated based on the first order cumulant expansion formula, the second order cumulant expansion formula, and the full exponential formula. The convergence of the free energy curves derived from the second order cumulant and the full exponential formula was used as an indication that the work distribution is 
Gaussian. ${ }^{29,30}$ For the regions corresponding to poor convergence of the two curves, further equilibration was performed for $1 \mathrm{~ns}$ under constrained MD conditions, and 20 snapshots were taken every 50 ps as the initial conditions for SMD runs with the same velocity as that used for the previous runs ( $\AA \AA / \mathrm{ns})$. The free energy analysis was then performed on the data collected from 40 trajectories for the same pulling length. This did not improve the convergence of the free energy curves derived from the second order cumulant and the full exponential formula. However, when the velocity of pulling was lowered to onequarter of the original velocity $(1.25 \AA / n s)$, the convergence improved significantly. Hence the error, which was a systematic one arising from the non-Gaussian behavior of the probability densities of work, was decreased significantly by pulling with a lower velocity. The calculation in the regions where the convergence was good at the original pulling velocity was not repeated.

To study the behavior of the uncapped ring at the nonane/ water interface, a box containing 126 nonane molecules and 1598 water molecules was created. The box was equilibrated for $1 \mathrm{~ns}$ using the NPT ensemble, allowing isotropic cell fluctuations. After a few picoseconds, the cell dimensions converged to about $41.5 \times 42.5 \times 49 \AA$. The equilibration was followed by two 4 ns simulations: one starting with the entire uncapped peptide ring immersed in water and the other inside nonane. During these simulations, the dimensions of the unit cell were kept constant in the $x-y$ plane while allowing fluctuations along the $z$-axis (the interfacial direction) using Langevin piston pressure control with a pressure of $1 \mathrm{~atm}$.

\section{Results and Discussion}

The present MD simulations indicate that the uncapped and the capped dimers stay together by means of intersubunit hydrogen bonds in nonpolar solvent, whereas they fall apart in polar solvent. Figure 1a shows an uncapped ring, while Figure $1 \mathrm{~b}$ shows a capped ring. Figure $1 \mathrm{c}, \mathrm{d}$ shows the uncapped dimer in nonane after $1 \mathrm{~ns}$ and $10 \mathrm{~ns}$ of MD simulations, respectively. Figure 1e,f shows the uncapped dimer in water at the end of 1 $\mathrm{ns}$ and $10 \mathrm{~ns}$ of MD, respectively. In both the cases, the C $\alpha$ atoms of the backbones were fixed to their initial positions during the first nanosecond of MD. Cases I and III involve capped dimers in polar and nonpolar solvents, respectively, and correspond to systems accessible experimentally. Here, the present results are consistent with the experimental data. Simulation cases II and IV represent pure dimeric systems that are inaccessible to experiment: there are only two peptide rings in the simulation unit cell. Experimentally, when self-assembly occurs, not only would the dimer form, but the trimer and longer assemblies would also be present. The ${ }^{1} \mathrm{H}$ NMR and IR studies by Ghadiri et al. ${ }^{5}$ showed that cyclo [(-L-Phe-D-N-MeAla- $\left.4_{4}-\right]$ displays several conformational isomers in polar solvents, such as deuterated methanol or dimethyl sulfoxide (DMSO), which slowly interconvert as a result of the cis-trans isomerization of secondary amides. This inhibits the dimerization process in polar solvent for the capped dimer. The factors that prevent the dimerization of the capped subunit in polar solvent should also inhibit the dimerization of the uncapped subunit in polar solvent. Experimental studies have confirmed the self-assembly of the peptide subunit studied here in a lipid bilayer environment. This leads us to predict that the uncapped dimer should be stable in a nonpolar solvent while being unstable in a polar solvent. Our results agree with this prediction.

Experimental studies have indicated that the peptide nanotubes and the dimer formed by the self-assembly of cyclic peptide rings possess a $\beta$-sheet structure. Figure $2 \mathrm{a}$ shows the Ramachandran plot obtained from the MD simulation of a single uncapped peptide ring in nonane. This plot clearly shows that the $\varphi$ and $\psi$ angles of all the residues lie in the $\beta$-sheet region. The signs of the $\varphi$ 's and $\psi$ 's for the D-amino acids are simply the inverse of what they should be for the L-amino acids. This tells us that the geometry of the single peptide ring dictates the preference for a $\beta$-sheet structure, even when it is not hydrogen bonded to another subunit. Figure $2 b$ shows the radius of gyration of the backbone atoms of the ring versus time. The ring has a flat conformation in the beginning of the simulation. The radius of gyration stays close to the initial value throughout the simulation, indicating the ring stays flat in nonane.

Figure 3 shows the time evolution of the number of hydrogen bonds between the subunits constituting the dimer under different conditions, following $1 \mathrm{~ns}$ of constrained MD. Concerning the definition of a hydrogen bond, we say a hydrogen bond exists if the distance between the donor and acceptor atoms is less than $3.4 \AA$ and the angle $\mathrm{D}-\mathrm{H}-\mathrm{A}$ is greater than $130^{\circ}$, where $\mathrm{D}$ represents the donor atom, $\mathrm{H}$ represents the hydrogen atom and $\mathrm{A}$ represents the acceptor atom. The number of hydrogen bonds shown is the average number of hydrogen bonds calculated every $100 \mathrm{ps}$. The maximum possible number of hydrogen bonds between the two subunits is eight. As shown in the figure, the number of intersubunit hydrogen bonds remains close to eight for the uncapped dimer in nonane. Although the number of hydrogen bonds for the capped dimer in nonane fluctuates more than for the uncapped dimer in nonane, at least six intersubunit hydrogen bonds always exist between the two rings during the entire $9 \mathrm{~ns}$ length of the trajectory. The number of hydrogen bonds during the initial $1 \mathrm{~ns}$ of constrained MD is not plotted in the figure, as it is expected to be eight for all the systems. The quantum mechanical studies by Chen et al. ${ }^{10}$ showed that the conformation for the optimized cyclo [(-L-PheD-N-MeAla- $)_{4}$ - is distorted to a certain extent compared to that for cyclo [(-L-Phe-D-Ala- $\left.)_{4}-\right]$. It was observed that the angles of $\mathrm{NH}$ and $\mathrm{CO}$ with respect to the ring plane in the capped ring depart farther than that found for the uncapped ring. A departure of $\pm 10^{\circ}$ from the normal value of $180^{\circ}$ for $\omega$ was also observed. Thus, distortion of the capped ring compared to that of the uncapped ring could be the possible reason for the slight reduction in the number of intersubunit hydrogen bonds for the capped dimer versus the uncapped dimer. Figure 3 also shows that the number of hydrogen bonds between the two rings falls to zero after about $1 \mathrm{~ns}$ of unconstrained MD for the capped dimer in water. Although it takes much longer for the hydrogen bonds to break between the two monomers of the uncapped dimer in water, the monomeric state is more stable as the dimer breaks at the end of $10 \mathrm{~ns}$. We stop the simulation at the end of $10 \mathrm{~ns}$, even though the two rings have not completely fallen apart, as the breaking of the remaining hydrogen bonds is just a matter of running for more time. One possible reason for the faster breaking of the intersubunit hydrogen bonds in the capped dimer, compared to those in the uncapped dimer, could be the conformational distortion of the capped ring compared to the uncapped ring as explained previously. The exposure of the hydrophobic methyl groups of the capped dimer to the hydrophilic water molecules could be another reason for the higher instability of the capped dimer. Figure 4 shows the number of hydrogen bonds between the backbone atoms of the peptide rings and the water molecules using the same definition of the hydrogen bond as previously defined for intersubunit hydrogen bonds. Here again, the number of hydrogen bonds shown is the average number of hydrogen bonds calculated every 100 


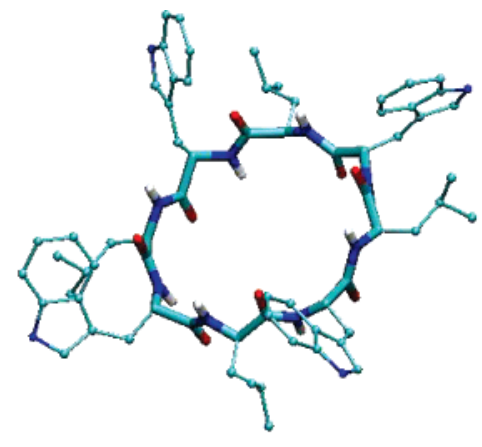

a

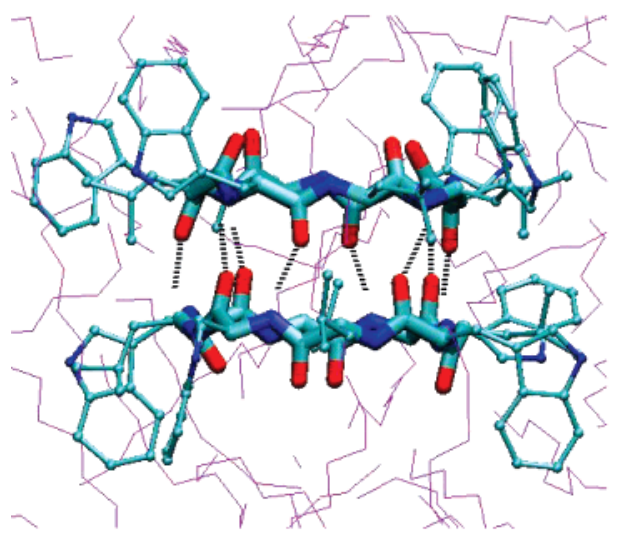

c

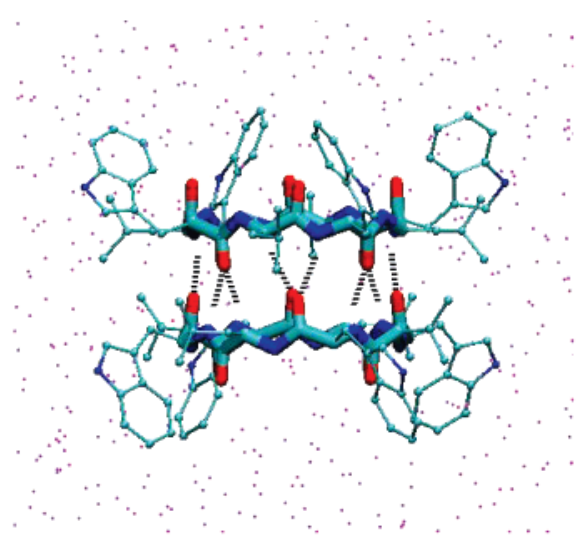

e

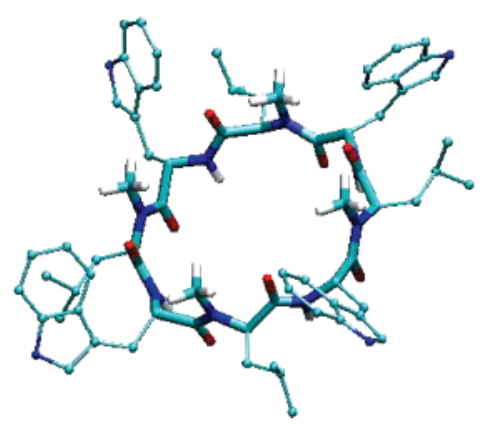

b

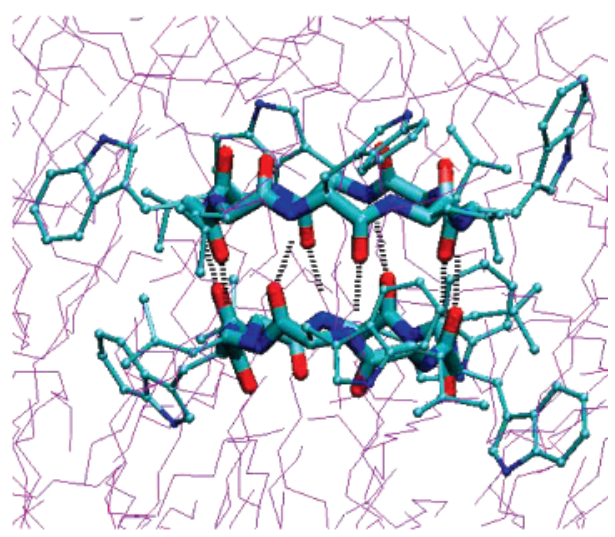

d

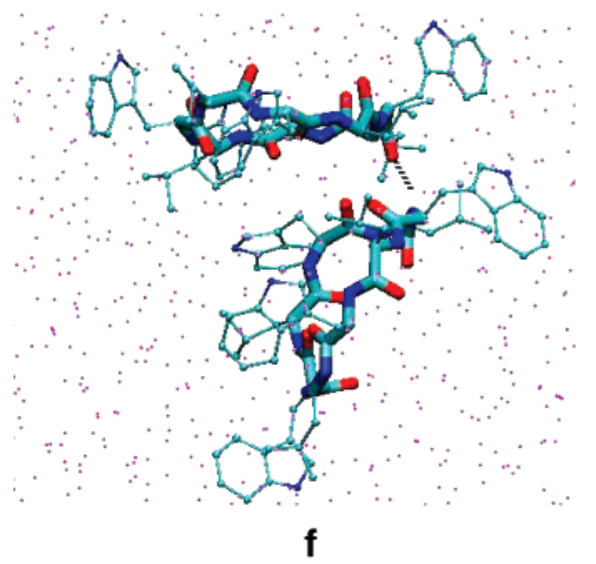

Figure 1. Representative configurations taken from MD simulations of cyclic peptide dimers: (a) one uncapped ring; (b) one capped ring. Panel a shows the hydrogen atoms of the $\mathrm{N}-\mathrm{H}$ groups of the backbone, while panel $\mathrm{b}$ shows the hydrogen atoms of the Leu $\mathrm{N}-\mathrm{H}$ groups substituted by methyl groups. (c) The uncapped dimer in nonane at $t=1 \mathrm{~ns}$. (d) The uncapped dimer in nonane at $t=10 \mathrm{~ns}$. Panels c and d show that the planar rings stay close to each other in the nonpolar solvent. Intersubunit hydrogen bonds contribute to the stability of the dimer. (e) The uncapped dimer in water at $t=1 \mathrm{~ns}$. (f) The uncapped dimer in water at $t=10 \mathrm{~ns}$. Panel e shows that the rings exist in the form of a dimer after $1 \mathrm{~ns}$ of constrained MD during which the backbone $\mathrm{C} \alpha$ atoms are fixed, while panel $\mathrm{f}$ shows that the intersubunit hydrogen bonds break at the end of $10 \mathrm{~ns}$. Carbon atoms are green, oxygen atoms are red, and nitrogen atoms are blue. The cyclic peptide backbones are rendered as tubes, and the side chains are shown as stick and ball. The solvent molecules are shown in purple. The hydrogen bonds between the two rings are shown as dashed black lines. In panels $\mathrm{a}$ and $\mathrm{b}$, only the hydrogen atoms of the $\mathrm{N}-\mathrm{H}$ groups of the backbones are shown; in all other cases, all hydrogen atoms have been omitted for clarity. The images were made using VMD. ${ }^{33}$

ps. In the case of capped dimer, only the uncapped side of the ring can participate in hydrogen bonding with water, whereas, for the uncapped ring, both faces of the ring can take part in hydrogen bonding with water. However, we measure the number of hydrogen bonds formed only by that side of the ring that takes part in intersubunit hydrogen bonding. This gives eight hydrogen-bonding sites on each ring: four $\mathrm{N}-\mathrm{H}$ sites and four $\mathrm{C}-\mathrm{O}$ sites. Each backbone oxygen atom can participate in two hydrogen bonds with water molecules. Hence, each ring can form a maximum of 12 hydrogen bonds with water if all the sites participate in hydrogen bonding with water. From Figures 3 and 4 we can clearly see that, as the number of intersubunit hydrogen bonds goes down, the number of hydrogen bonds with water increases for both the capped and the uncapped dimers in water.

The competition between intersubunit hydrogen bonding and hydrogen bonding with the solvent has also been discussed in another study. ${ }^{31}$ In that study, it was observed that, under rigorously anhydrous conditions, the association constant for cyclo [(-L-Phe-D-N-MeAla- $\left.)_{4}-\right]_{2}$ in deuteriochloroform approxi- 

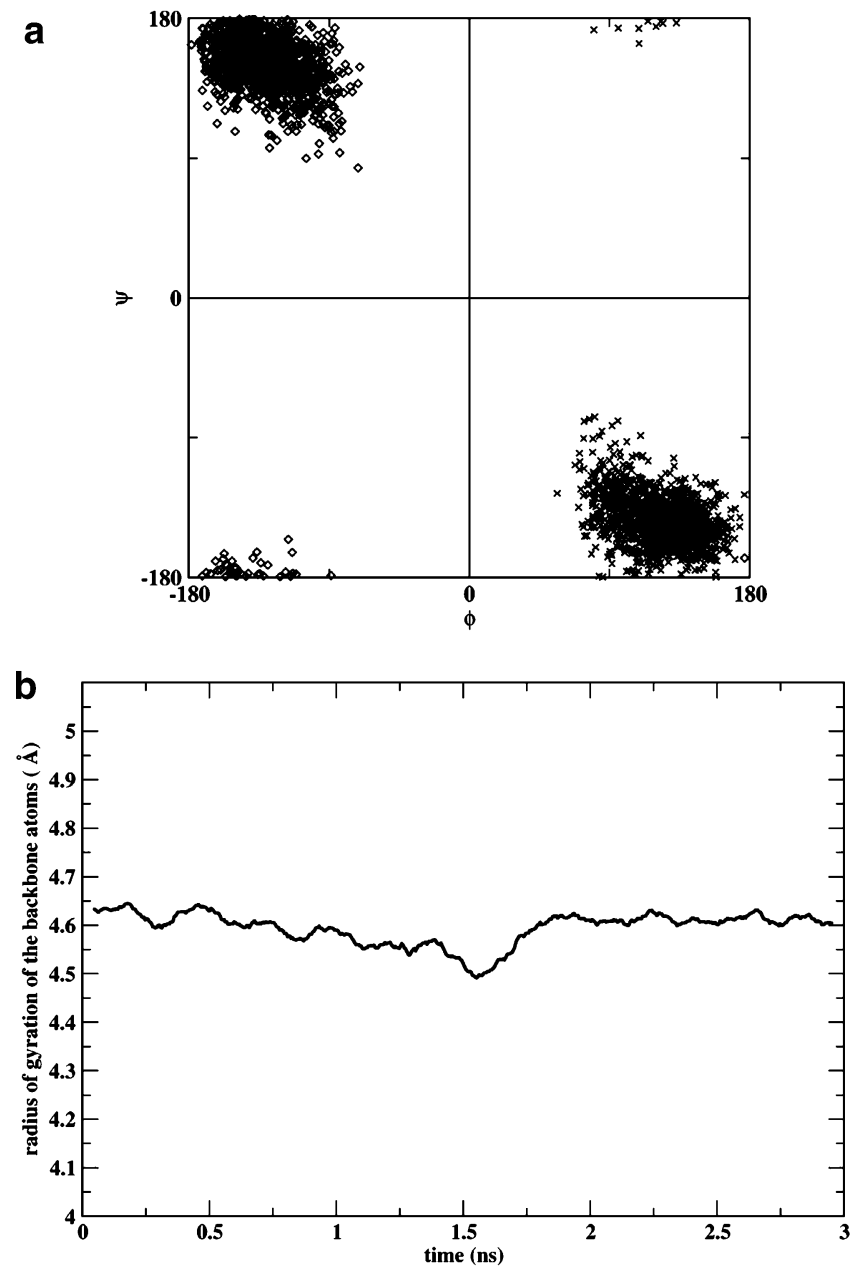

Figure 2. (a) Ramachandran plot for a single uncapped peptide ring in nonane. The dihedrals for L-Trp residues are shown with diamondshaped symbols, while the dihedrals for D-Leu residues are shown with crosses. The plot clearly shows that the ring has a $\beta$-sheet structure. (b) Radius of gyration of the backbone atoms of one uncapped ring in nonane. The radius of gyration stays close to the initial value throughout the simulation, indicating that the ring stays in a flat conformation in nonane. The range of the $y$ axis is chosen for easy comparison with Figure 5.

mately doubled, suggesting competition by water for intermolecular hydrogen-bonding sites. Weak low-energy bands were observed in FT-IR spectra of peptides that do not form dimers, presumably arising from intramolecular hydrogen bonding.

We measured the distance between the centers of mass of the backbone atoms of the two rings. The average intersubunit distance over the last $5 \mathrm{~ns}$ was found to be $4.78 \pm 0.08 \AA$ for the uncapped dimer in nonane and $4.96 \pm 0.10 \AA$ for the capped dimer in nonane. These values agree well with the values of 4.7-4.8 $\AA$ obtained by electron diffraction patterns, X-ray analyses, and IR measurements. ${ }^{2,32}$ On the other hand, the average intersubunit distance calculated during the last $5 \mathrm{~ns}$ of the MD simulations was measured to be about $6.4 \AA$ for the uncapped dimer in water and $10.93 \AA$ for the capped dimer in water.

Thus, the simulations demonstrate that both the capped and the uncapped dimers remain stable in a hydrophobic solvent. This result agrees with the ${ }^{1} \mathrm{H}$ NMR and IR results obtained by Ghadiri et al. ${ }^{5}$ for the capped dimer. They observed that cyclo [(-L-Phe-D-N-MeAla-) $\left.4^{-}\right]$displays several conformational isomers in polar solvents such as deuterated methanol or DMSO, which slowly interconvert as a result of cis-trans isomerization. However, in nonpolar solvents such as carbon tetrachloride

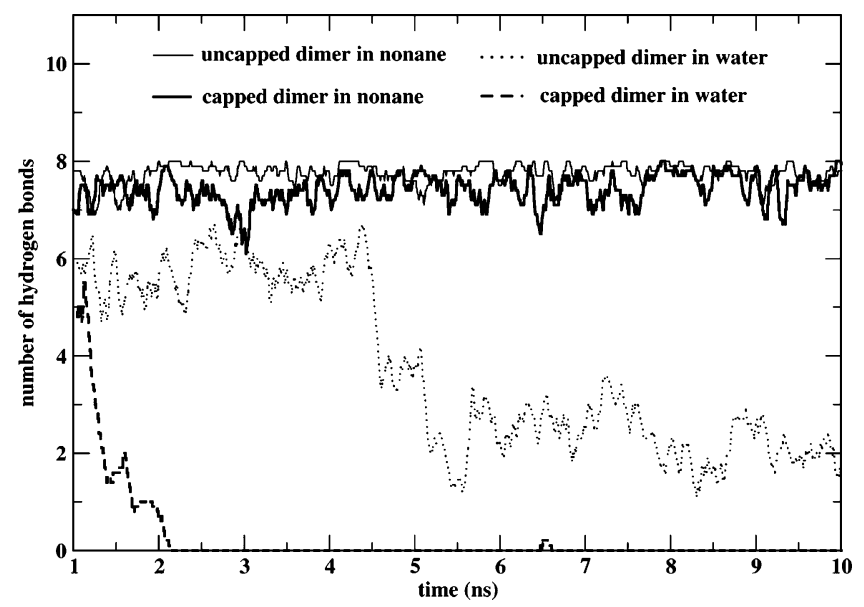

Figure 3. Time evolution of the average number of intersubunit hydrogen bonds in the four MD simulations. A hydrogen bond exists between two atoms if the distance between the donor and acceptor atoms is less than $3.4 \AA$ and the angle $\mathrm{D}-\mathrm{H}-\mathrm{A}$ is greater than $130^{\circ}$, where $\mathrm{D}$ represents the donor atom, $\mathrm{H}$ represents the hydrogen atom, and $\mathrm{A}$ represents the acceptor atom. The figure shows that the number of hydrogen bonds remains almost constant for both the uncapped dimer and the capped dimer in nonane. All the hydrogen bonds break after about $2 \mathrm{~ns}$ for the capped dimer in water, while it takes about $4.5 \mathrm{~ns}$ for most of the intersubunit hydrogen bonds to break for the uncapped dimer in water. The number of hydrogen bonds during the first nanosecond of constrained MD is not shown.

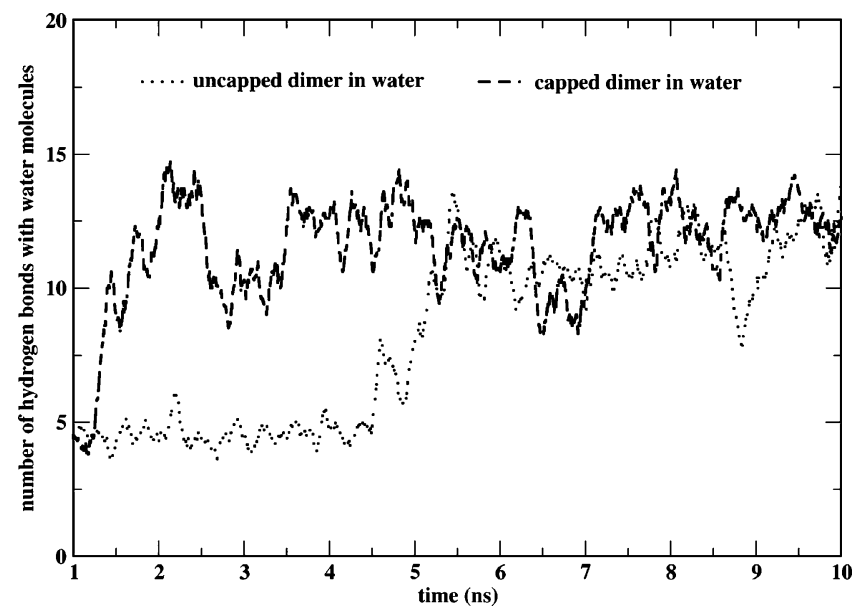

Figure 4. Hydrogen bonds between water molecules and peptide rings. Upon comparing Figures 3 and 4, we can clearly see that, as the number of intersubunit hydrogen bonds decreases, the number of hydrogen bonds with water molecules increases. The definition of hydrogen bonds used is the same as that for intersubunit hydrogen bonds.

$\left(\mathrm{CCl}_{4}\right)$, the peptide exists in an all-trans conformation with a flat-ring-shaped backbone, which is in dynamic equilibrium with the expected cylindrical dimer. Regarding the uncapped dimer, as discussed previously, we expected the dimer to be stable in nonpolar solvent because a nanotube made of the peptide subunit is stable in a lipid bilayer. Our observation here agrees with this prediction. Figure 5 shows the radius of gyration of the backbone atoms of the ring versus time. The radius of gyration should be the maximum for the ring in a flat conformation. As seen clearly in the figure, the radius of gyration stays close to the initial value, corresponding to a flat conformation for both the capped and the uncapped dimers in nonane. In Figure 2b, it was seen that the backbone of a single uncapped ring also stays in a flat conformation in nonane. Figure 5 shows that the ring does not stay flat in water after the intersubunit hydrogen bonds break. Hence our results agree with the experimental results. 


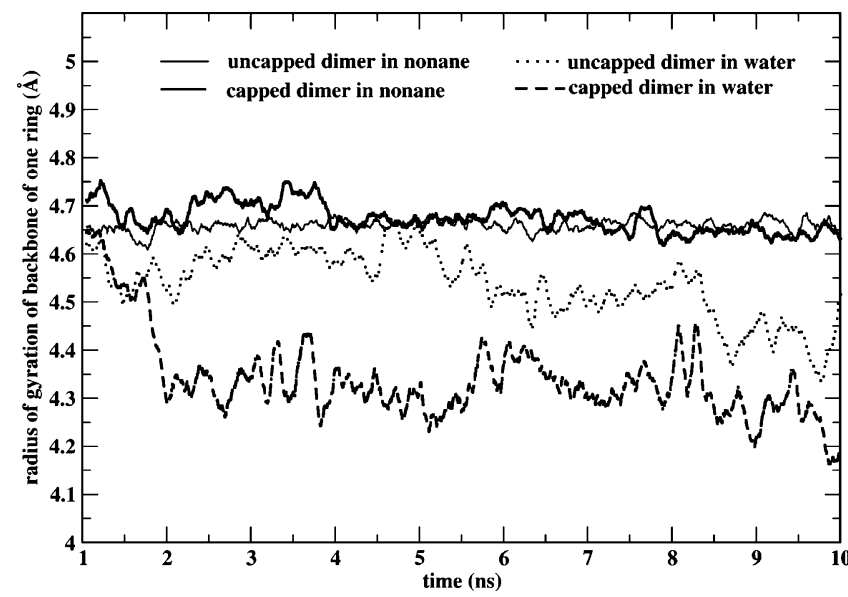

Figure 5. Radius of gyration of the backbone atoms of one ring of the dimer studied under four different conditions. The radius of gyration should be the maximum, corresponding to a flat conformation of the ring. It is clear from the figure that, as the intersubunit hydrogen bonds break and the rings separate from each other in water, the radius of gyration decreases and the ring does not stay in a flat conformation.

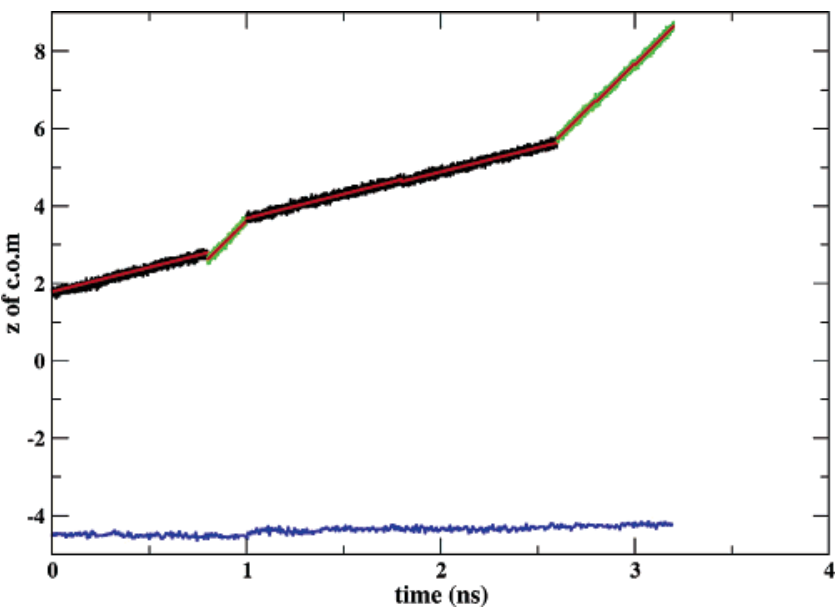

Figure 6. The $z$ coordinates of the centers of mass (c.o.m) of the two rings vs time. The blue line shows the $z$ coordinate of the fixed ring 1 . The red line shows the position of the dummy atom attached to the $z$ coordinate of the pulled ring 2 (reaction coordinate). The $z$ trajectory of the c.o.m of ring 2 (pulling coordinate) is shown in black for regions where the velocity of pulling was $1.25 \AA / n$ s and green for regions where the velocity of pulling was $5 \AA / n s$. The complete pulling trajectory was divided into seven sections. Each section consisted of 20 pulling runs. Data from only one trajectory for each section are shown as an example. As seen from the figure, the pulling coordinate closely follows the reaction coordinate for ring 2 .

Figure 6 shows the $z$ coordinate of the center of mass of the fixed ring 1 versus time and the $z$ coordinate of the center of mass of the pulled ring 2 versus time. As seen in the figure, the $z$ coordinate of ring 2 closely follows the reaction coordinate, that is, the $z$ coordinate of the dummy atom attached to it. As explained in the simulation details, the whole pulling trajectory was divided into seven sections, each consisting of 20 pulling runs. The plots shown in Figure 6 are only for one pulling run chosen independently for each section. The free energy profile of the distance between the two capped rings in nonane as a function of the $z$ coordinate of the pulled ring is shown in Figure 7. As explained in the simulation details, for certain sections, 40 pulling runs were performed and/or lower pulling velocities were used. Plots showing the effect of the number of pulling runs and the velocities on the free energy profile are available in the Supporting Information for the interested reader. The

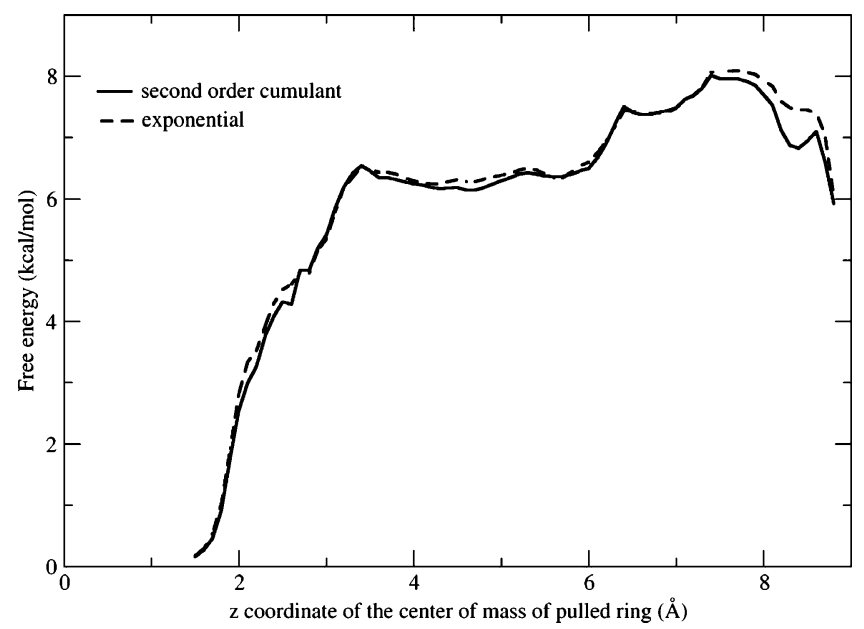

Figure 7. Change in free energy associated with pulling one capped ring away from the other capped ring of the stable dimer in nonane. The free energy is calculated by using the second-order cumulant approximation of Jarzynski's equality and the full exponential formula.
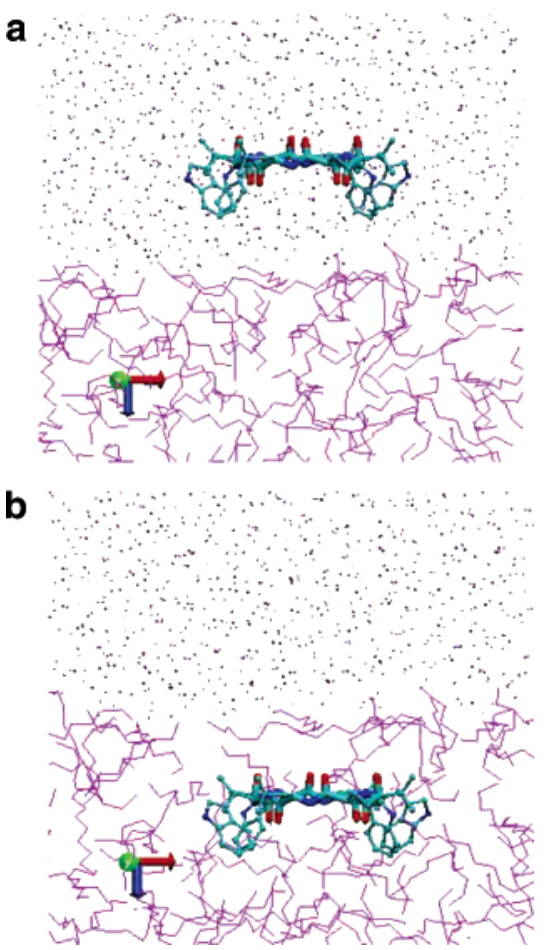

Figure 8. (a) Starting configuration for the uncapped peptide ring in the water phase and (b) starting configuration for the uncapped peptide ring in the nonane phase. Carbon atoms are green, oxygen atoms are red, and nitrogen atoms are blue. The cyclic peptide backbones are rendered as tubes, and the side chains are shown as stick and ball. Nonane molecules are shown as thin purple lines, and water molecules are shown as purple spheres. Hydrogen atoms have been omitted for clarity. The images were made using VMD..$^{33}$

availability of experimental data for a capped dimer was the reason for our choice of the capped dimer for the free energy calculation, although both the capped dimer and the uncapped dimer are stable in nonane. The initial position of the pulled ring corresponds to a distance of $\sim 6.5 \AA$ between the $z$ coordinates of the centers of mass of the two rings. After pulling for about $2 \AA$, the backbones of the two rings stop interacting with each other. All the intersubunit hydrogen bonds are broken at this point. Further changes in the free energy occur as a result of the formation and disruption of hydrogen bonds between the Trp side chains of one ring (either ring 1 or ring 2) and the 
a

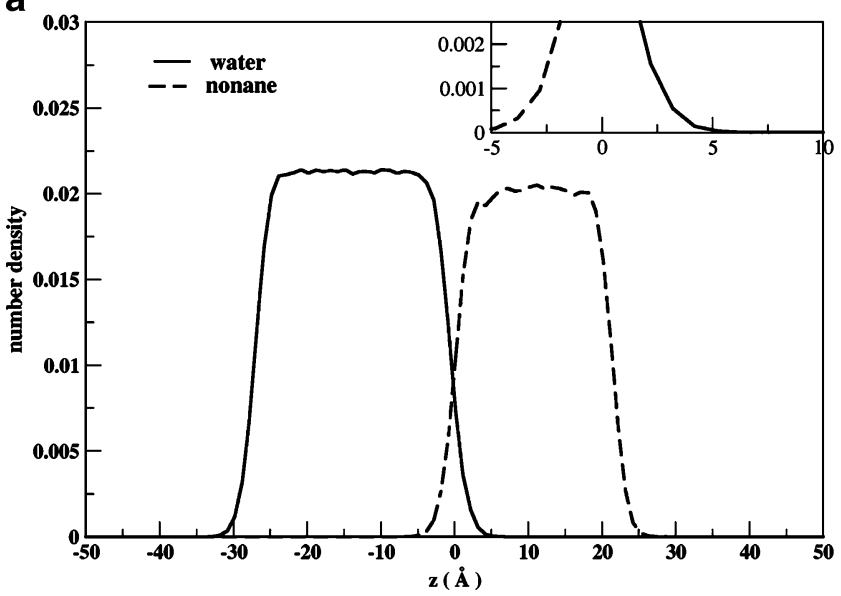

b

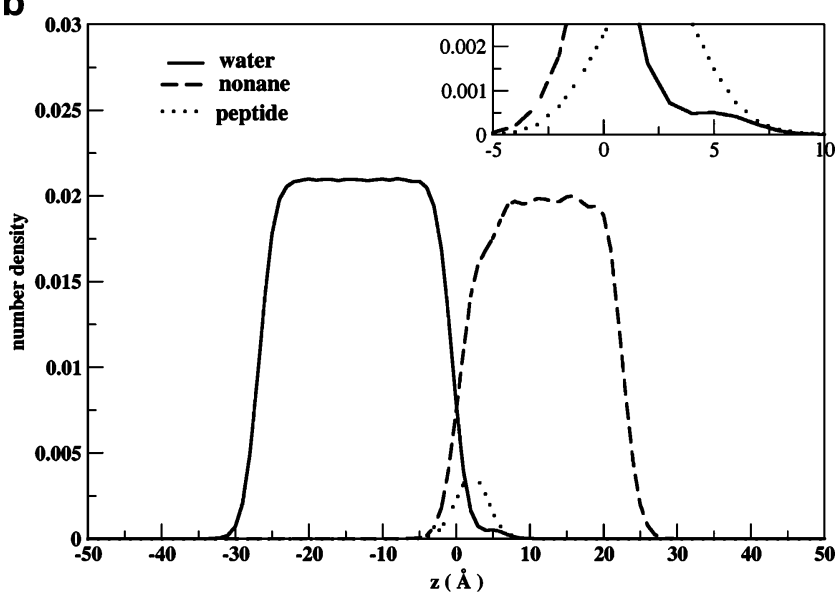

C

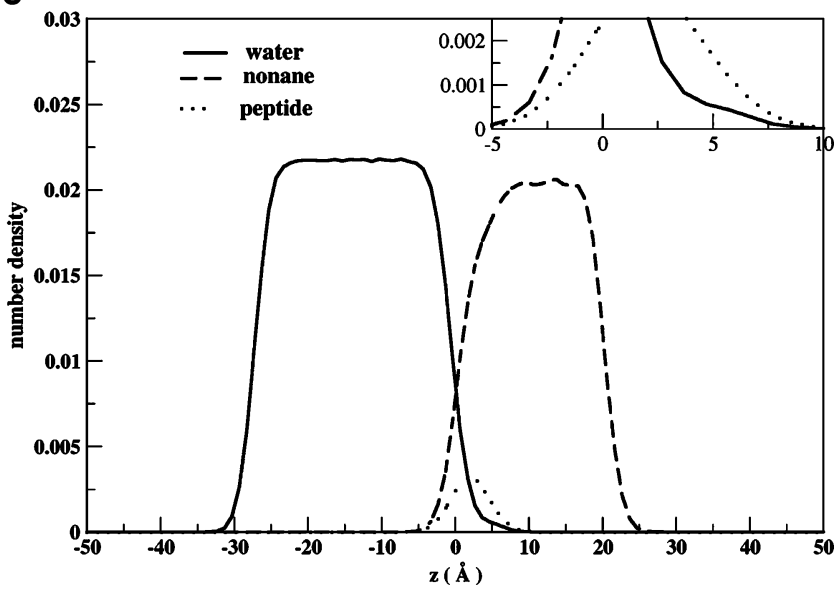

Figure 9. Number density profiles for the different components of the system along the normal to the nonane/water interface: (a) the pure nonane/water interface; (b) the density profiles obtained from the last $2 \mathrm{~ns}$ of the $4 \mathrm{~ns}$ simulation corresponding to the initial condition of a single uncapped peptide ring completely immersed in the water phase; and (c) the density profiles obtained from the last $2 \mathrm{~ns}$ of the $4 \mathrm{~ns}$ simulation corresponding to the initial condition of a single uncapped peptide ring completely immersed in the nonane phase. As seen clearly from panels $\mathrm{b}$ and $\mathrm{c}$, the ring prefers to reside close to the interface in the nonane phase, irrespective of the initial configuration. Also, more water molecules penetrate the nonane phase in panels $b$ and c compared to those in panel a (see the insets). These water molecules are associated with the hydrophilic sites on the peptide ring via hydrogen bonds.

backbone of the other ring. A figure showing the intersubunit hydrogen bonds during the pulling simulation is available in the Supporting Information for the interested reader. The free energy difference between the initial state of the two rings when they are hydrogen bonded to each other and when they are separated is $\sim 7 \pm 1 \mathrm{kcal} / \mathrm{mol}$, derived from the second order cumulant approximation and the full exponential formula. This result is reasonable, given the value of $4.0-5.6 \mathrm{kcal} / \mathrm{mol}$ obtained by Ghadiri et al., ${ }^{5}$ with a dimer made up of different amino acid residues and a different solvent. As mentioned by Kobayashi et al., ${ }^{21}$ it should be expected that the changes in the polarity of the medium may produce significant and additive energetic contributions, emanating from cross-strand interactions between side chains, toward stabilizing a particular $\beta$-sheet arrangement.

To further understand the process of self-assembly of peptide rings in a lipid bilayer environment, we studied the behavior of a single ring in a box with a nonane/water interface. Figure 9a shows the number density profiles obtained from a 1 ns simulation of a pure nonane/water interface along the direction normal to the interface ( $z$ direction). A single uncapped peptide ring was immersed completely in the water phase with the center of the peptide backbone at a distance of about $6 \AA$ from the nonane phase, as shown in Figure 8a. Figure $9 \mathrm{~b}$ shows the density profiles obtained from the last $2 \mathrm{~ns}$ of the $4 \mathrm{~ns}$ MD simulation of this system along the $z$ direction. In another simulation, a single uncapped peptide ring was immersed completely in the nonane phase with the center of the peptide backbone at a distance of about $9 \AA$ from the water phase as shown in Figure 8b. Figure 9c shows the density profiles obtained from the last $2 \mathrm{~ns}$ of the 4 ns MD simulation of this system. From Figures $9 \mathrm{~b}$ and $9 \mathrm{c}$ it is clear that the peptide ring prefers to sit close to the interface in the nonane phase, irrespective of the initial configuration. This observation can be explained as a result of the amphiphilic nature of the peptide ring. This tells us that a single peptide ring in a lipid bilayer environment should prefer to sit close to the hydrophobic/ hydrophilic interface. However, the hydrophobic/hydrophilic interface in a hydrated lipid bilayer is not as well-defined as a pure nonane/water interface. It can be said from our simulation results that the peptide ring behaves like a surfactant at a nonane/ water interface. The lipid molecules also possess surfactant properties, with a hydrophilic headgroup and a hydrophobic tail. The behavior of the peptide ring in the presence of another surfactant molecule in a lipid bilayer might be different than its behavior at a pure hydrophobic/hydrophilic interface.

Upon comparing Figure $9 a-c$, we can clearly see that the water phase extends deeper into the nonane phase in panels $b$ and c compared to that in panel a. These water molecules entering the nonane phase are associated with the hydrophilic sites on the peptide ring by hydrogen bonding.

\section{Conclusions}

This study characterizes the behavior of the dimers formed from cyclo $\left[(-\mathrm{L}-\mathrm{Trp}-\mathrm{D}-\mathrm{Leu})_{4}\right]_{2}$ and cyclo [(-L-Trp-D-NMeLeu- $\left.)_{4}-\right]_{2}$ in polar and nonpolar environments under the CHARMM force field. The observed stability of the dimers and the flat conformation of the peptide ring in a hydrophobic solvent matches well with the experimental results. The dimers are observed to be unstable in a hydrophilic solvent. We also observe that a single peptide ring prefers to sit close to the nonane/water interface. This suggests that the self-assembly of the peptide rings to form a nanotube starts after the rings enter the hydrophobic tail region of a lipid bilayer. We also show that the $\varphi$ and $\psi$ angles of a single uncapped peptide ring in nonane lie in the $\beta$-sheet region of the Ramachandran plot. The potential of mean force was computed as a function of the 
distance between the two rings using SMD and Jarzynski's equality. This simulation study is a necessary prelude to the assembly of the complete nanotube. The understanding of the mechanism of the formation of these nanotubes will likely cast light on their mode of action as antibacterial agents and on other applications such as molecular inclusion devices and drugdelivery agents.

Acknowledgment. The authors thank M. Reza Ghadiri for providing the coordinates for the dimer and Ivaylo Ivanov for useful discussions. We acknowledge the Pittsburgh Supercomputing Center through the Pennsylvania research initiative for computer time used for these simulations. This work was funded by the National Institute of Health, Grant GM 40712.

Supporting Information Available: Plots showing the effect of the number of pulling runs and the velocities on free energy profiles and a figure showing the intersubunit hydrogen bonds during the pulling run. This material is available free of charge via the Internet at http://pubs.acs.org.

\section{References and Notes}

(1) Lopez, S. F.; Kim, H. S.; Choi, E. C.; Delgado, M.; Granja, J. R.; Khasanov, A.; Kraehenbuehl, K.; Long, G.; Weinberger, D. A.; Wilcoxen, K. M.; Ghadiri, M. R. Nature 2001, 412, 452.

(2) Ghadiri, M. R.; Granja, J. R.; Milligan, R. A.; McRee, D. E.; Khazanovich, N. Nature 1993, 366, 324

(3) Kim, H. S.; Hartgerink, J. D.; Ghadiri, M. R. J. Am. Chem. Soc. 1998, 120, 4417.

(4) Ghadiri, M. R.; Granja, J. R.; Buehler, L. K. Nature 1994, 369, 301 .

(5) Ghadiri, M. R.; Kobayashi, K.; Granja, J. R.; Chadha, R. K.; McRee, D. E. Angew. Chem., Int. Ed. Engl. 1995, 34, 93.

(6) Ghadiri, M. R. Adv. Mater. 1995, 7, 675.

(7) Hartgerink, J. D.; Clark, T. D.; Ghadiri, M. R. Chem.-Eur. J. 1998, 4, 1367 1570 .

(8) Carloni, P.; Andreoni, W.; Parinello, M. Phys. Rev. Lett. 1997, 106,

(9) Lewis, J. P.; Pawley, N. H.; Sankey, O. F. J. Phys. Chem. B. 1997, 101,10576
(10) Chen, G.; Su, S.; Liu, R. J. Phys. Chem. B. 2002, 106, 1570.

(11) Engels, M.; Bashford, D.; Ghadiri, M. R. J. Am. Chem. Soc. 1995, 117,9151

(12) Tarek, M.; Maigret, B.; Chipot, C. Biophys. J. 2003, 85, 2287.

(13) Quesada, J. S.; Isler, M. P.; Ghadiri, M. R. J. Am. Chem. Soc. 2002 124,10004

(14) Quesada, J. S.; Kim, H. S.; Ghadiri, M. R. Angew. Chem., Int. Ed. Engl. 2001, 40, 2503

(15) Motesharei, K.; Ghadiri, M. R. J. Am. Chem. Soc. 1997, 119, 11306

(16) Crooks, G. E. Phys. Rev. E 1999, 60, 2721

(17) Collin, D.; Ritort, F.; Jarzynski, C.; Smith, S. B.; Tinoco, I., Jr.; Bustamante, C. Nature 2005, 437, 231.

(18) Jarzynski, C. Phys. Rev. Lett. 1997, 78, 2690

(19) Jensen, M. O.; Park, S.; Tajkhorshid, E.; Schulten, K. Proc. Natl. Acad. Sci. U.S.A. 2002, 99, 6731.

(20) Amaro, R.; Tajkhorshid, E.; Schulten, Z. L. Proc. Natl. Acad. Sci. U.S.A. 2003, 100, 7599.

(21) Kobayashi, K.; Granja, J. R.; Ghadiri, M. R. Angew. Chem., Int. Ed. Engl. 1995, 34, 95.

(22) Kale, L; Skeel, R.; Bhandarkar, M.; Brunner, R.; Gursoy, A.; Krawetz, N.; Phillips, J.; Shinozaki, A.; Varadarajan, K.; Schulten, K. J. Comput. Phys. 1999, 151, 283.

(23) MacKerell, A. D.; Bashford, D., Jr.; Bellott, M.; Dunbrack, R. L.; Evanseck, J. D., Jr.; Field, M. J.; Fischer, S.; Gao, J.; Guo, H.; Ha, S.; Joseph-McCarthy, D.; Kuchnir, L.; Kuczera, K.; Lau, F. T. K.; Mattos, C.; Michnick, S.; Ngo, T.; Nguyen, D. T.; Prodhom, B.; Reiher, W. E.; Roux, B., III; Schlenkrich, M.; Smith, J. C.; Stote, R.; Straub, J.; Watanabe, M.; Wiórkiewicz-Kuczera, J.; Yin, D.; Karplus, M. J. Phys. Chem. B. 1998, 102,3586

(24) Ryckaert, J. P.; Ciccotti, G.; Berendson, H. J. C. J. Comput. Phys 1977, 23, 327.

(25) Andersen, H. C. J. Comput. Phys. 1983, 52, 24.

(26) Darden, T.; York, D.; Pederson, L. J. Chem. Phys. 1993, 98, 10089

(27) Essmann, U.; Perera, L.; Berkowitz, M. L.; Darden, T.; Lee, H.; Pederson, L. G. J. Chem. Phys. 1995, 103, 8577.

(28) Jorgensen, W. L.; Chandrasekhar, J.; Madura, J. D.; Impey, R. W.; Klein, M. L. J. Chem. Phys. 1983, 79, 926.

(29) Park, S.; Khalilli-Araghi, F.; Tajkhorshid, E.; Schulten, K. J. Chem. Phys. 2003, 119, 3559.

(30) Hummer, G. J. Chem. Phys. 2001, 114, 7330

(31) Clark, T. D.; Buriak, J. M.; Kobayashi, K.; Isler, M. P.; McRee, D. E.; Ghadiri, M. R. J. Am. Chem. Soc. 1998, $120,8949$.

(32) Hartgerink, J. D.; Granja, J. R.; Milligan, R. A.; Ghadiri, M. R. J Am. Chem. Soc. 1996, 118, 43.

(33) Humphrey, W.; Dalke, A.; Schulten, K. J. Mol. Graphics 1996, $14,33$. 